\title{
Background Characterization for Thermal Ion Release Experiments with ${ }^{224} \mathbf{R a}$
}

\author{
Helen Kwong \\ Office of Science, Science Undergraduate Laboratory Internship (SULI) \\ Stanford University \\ Stanford Linear Accelerator Center \\ Menlo Park, California
}

August 19, 2005

Prepared in partial fulfillment of the requirements of the Office of Science, Department of Energy's Science Undergraduate Laboratory Internship under the direction of Peter Rowson in the Research Division at the Stanford Linear Accelerator Center.

Participant:

Signature

Research Advisor:

Signature 


\section{Table of Contents}

$\begin{array}{ll}\text { Abstract } & \text { ii }\end{array}$

1 Introduction $\quad 1$

2 Materials and Methods 3

3 Results $\quad 5$

3.1 In vacuum . . . . . . . . . . . . . . . . . . . . 5

3.2 In xenon gas . . . . . . . . . . . . . . . . . . . . . 5

$\begin{array}{lll}4 & \text { Discussion and Conclusion } & 7\end{array}$

4.1 Peak characterization . . . . . . . . . . . . . . . . 7

4.2 Time decay . . . . . . . . . . . . . . . . . . . . . . . . . 9

5 Acknowledgements $\quad 10$

6 Tables and Figures $\quad 11$

$\begin{array}{ll}\text { References } & 17\end{array}$ 


\begin{abstract}
Background Characterization for Thermal Ion Release Experiments with ${ }^{224}$ Ra. HELEN KWONG (Stanford University, Stanford, CA 94305) PETER ROWSON (Stanford Linear Accelerator Center, Menlo Park, CA 94025).
\end{abstract}

The Enriched Xenon Observatory for neutrinoless double beta decay uses ${ }^{136}$ Ba identification as a means for verifying the decay's occurrence in ${ }^{136} \mathrm{Xe}$. A current challenge is the release of $\mathrm{Ba}$ ions from the $\mathrm{Ba}$ extraction probe, and one possible solution is to heat the probe to high temperatures to release the ions. The investigation of this method requires a characterization of the alpha decay background in our test apparatus, which uses a ${ }^{228} \mathrm{Th}$ source that produces ${ }^{224} \mathrm{Ra}$ daughters, the ionization energies of which are similar to those of Ba. For this purpose, we ran a background count with our apparatus maintained at a vacuum, and then three counts with the apparatus filled with Xe gas. We were able to match up our alpha spectrum in vacuum with the known decay scheme of ${ }^{228} \mathrm{Th}$, while the spectrum in xenon gas had too many unresolved ambiguities for an accurate characterization. We also found that the alpha decays occurred at a near-zero rate both in vacuum and in xenon gas, which indicates that the rate was determined by ${ }^{228}$ Th decays. With these background measurements, we can in the future make a more accurate measurement of the temperature dependency of the ratio of ions to neutral atoms released from the hot surface of the probe, which may lead to a successful method of Ba ion release. 


\section{Introduction}

Two fundamental questions in neutrino physics are the determination of the neutrino masses, and whether the neutrino is its own antiparticle. Successful detection of neutrinoless doublebeta decay $(0 \nu \beta \beta)$ will allow us to measure the average neutrino mass, and establish the Majorana nature of neutrinos, a discovery that would also have profound implications for other areas of physics. However, thus far no experiment has been able to establish unambiguously that $0 \nu \beta \beta$ occurs. The Enriched Xenon Observatory (EXO) seeks to observe $0 \nu \beta \beta$ decays in ${ }^{136} \mathrm{Xe}$, and in order to verify the occurrence of double-beta decay, EXO will attempt to detect ions of ${ }^{136} \mathrm{Ba}$, the daughter nucleus of ${ }^{136} \mathrm{Xe}$. This involves using ionization and scintillation light to determine the event location, extracting the barium ion from the event site by a probe, and transporting it to a cell for laser spectroscopy analysis. Among the current challenges for the EXO experiment, now in the R\&D stage, is the release of the barium ion after its capture by the probe.

Since heated metal surfaces can release captured metal atoms in both the neutral or ionized state, one possible method of ion release is to heat the probe to high temperatures [1]. This method requires that the platinum surface is heated to a high enough temperature to liberate the barium ions, without releasing a significant proportion of neutral atoms. Therefore, the temperature dependency of the ratio of neutral to ionized atoms on a platinum surface has to be determined.

In our thermal ion release experiments, we will test a platinum surface with ${ }^{224} \mathrm{Ra}$ ions, since the first and second ionization energies of radium are similar to those of barium, according to [1]. Our ion source will be ${ }^{228} \mathrm{Th}$, which produces ${ }^{224}$ Ra daughters and has a decay scheme that leads eventually to a stable ${ }^{208} \mathrm{~Pb}$ (see Figure 1) [2]. The experiments will be conducted in a cell filled with xenon gas, and background sources must be taken into account. In this paper, we report on our characterization of the background, measured both 
with the cell in vacuum and with the cell filled with xenon gas. 


\section{Materials and Methods}

The main part of our system is a chamber built almost entirely out of UHV-compatible materials, and Figure 2 shows a diagram of the chamber. Inside the chamber, in the center, is a small piece of platinum foil mounted on two tungsten rods, which allow the foil to be moved up and down, and are connected to a power supply that we will use to heat the foil. Facing the foil is the ${ }^{224} \mathrm{Ra}$ source, with ${ }^{228} \mathrm{Th}$ electroplated onto a platinum disk, and it is connected to a high voltage power supply. Below the source is an $\alpha$ detector that is read out using standard pre- and post-amplification electronics. Between the source and the foil is a collimator plate; the plate has a hole in front of the detector, and is also connected to a high voltage power supply. Furthermore, the chamber is connected to a purifier that will reduce the impurities inevitably present in the system, and connected to the purifier is a turbomolecular pump.

In the actual thermal ion release experiments, the foil will receive the ions recoiled from the forward-biased source and then be heated by a current to temperatures over $1000 \mathrm{~K}$, to release the radium ions. The ion yield is determined by counting the $\alpha$ particles emitted in the ${ }^{224} \mathrm{Ra}$ decay, along with those produced by the subsequent $\alpha$ decays of ${ }^{220} \mathrm{Rn},{ }^{216} \mathrm{Po}$, ${ }^{212} \mathrm{Bi}$, and ${ }^{212} \mathrm{Po}$ (see Figure 1 ). We do not expect any detection of ${ }^{228} \mathrm{Th} \alpha$ decays, for the way the source is positioned above the detector makes it very unlikely that an $\alpha$ particle from a ${ }^{228}$ Th decay would be received by the detector.

For the first part of our background measurement, we pumped the cell down to a $5.0 \mathrm{x}$ $10^{-6}$ torr vacuum, had everything in the cell at zero voltage, and ran a background count for 68 hours. For the second part of the measurement, we pumped the cell down to a $1.7 \mathrm{x}$ $10^{-8}$ torr vacuum and then filled the vessel with research-grade xenon gas (which is quoted as $99.999 \%$ pure by the supplier) at about $0.5 \mathrm{~atm}$. The source was reversed-biased at -700 $\mathrm{V}$, the collimator plate was at $+500 \mathrm{~V}$, and the foil was biased at $+500 \mathrm{~V}$ with a temporary 
device. With this setup, in the ideal situation, the ${ }^{224} \mathrm{Ra}$ ions that resulted from ${ }^{228} \mathrm{Th}$ decays would have been immediately attracted back to the source. A count with the foil placed in front of the source was run for 72 hours, one with it in front of the detector, for 12 hours, and one with it in front of the stopper plate, for 12 hours (see Figure 2). Complicating our measurement was that we used a different post-amplifer for our xenon gas background counts, because the original one was malfunctioning. 


\section{Results}

\subsection{In vacuum}

Figure 3a shows a histogram of the pulse heights detected in vacuum. We can discern four clear peaks, and fitting them with Gaussian curves gives us mean values of $0.859 \mathrm{~V}, 0.957 \mathrm{~V}$, $1.039 \mathrm{~V}$, and $1.345 \mathrm{~V}$, as shown in Figure 3b. While the peak at $1.345 \mathrm{~V}$ is well isolated, the

other three are quite close to each other, with a shoulder between the two leftmost peaks. Restricting the data to the $0.8 \mathrm{~V}-1.1 \mathrm{~V}$ range and fixing the mean values of the three peaks, we do a Gaussian fit to obtain a mean of $0.922 \mathrm{~V}$ at the shoulder (Figure 3c).

Figure 4 shows a histogram of the times at which pulses were recorded. The two bursts of activity at approximately 160,000 s and 235,000 s were likely due to equipment heating that we performed at those times. Discounting those bursts by restricting the data to 140,000 s and below, we graph the time distribution for each of the four peak ranges of pulses and fit each to an exponential curve. We find that their fitted slopes are quite small and have relatively large errors (the slope would be $-k$ in a decay function $N(t)=N_{0} e^{-k t}$ ). The values are $7.01 \times 10^{-7} \pm 1.53 \times 10^{-6} \mathrm{~s}^{-1},-1.57 \times 10^{-6} \pm 1.37 \times 10^{-6} \mathrm{~s}^{-1}, 1.66 \times 10^{-6} \pm 1.33 \times 10^{-6}$ $\mathrm{s}^{-1}$, and $-2.12 \times 10^{-6} \pm 1.21 \times 10^{-6} \mathrm{~s}^{-1}$.

\subsection{In xenon gas}

As mentioned in Section 2, we did three background counts in xenon gas, each with the foil in a different position. Figure 5a shows the superimposition of the signal histograms for the three counts - red indicates the 72-hour count with the foil in front of the source, blue, the 12hour count in front of the detector, and green, the 12-hour count in front of the stopper plate ("discharge" position). The three histograms seem to exhibit the same patterns, showing peaks at similar positions, and doing Gaussian fits on the most obvious peaks for the 72-hour 
count (see Figure 5b) gives us mean values of $0.885 \mathrm{~V}, 0.962 \mathrm{~V}, 1.029 \mathrm{~V}, 1.144 \mathrm{~V}$, and $1.476 \mathrm{~V}$.

Figure 6 shows the time histograms for the xenon gas counts, each fitted to an exponential curve. The slopes for the source, detector, and discharge foil positions are respectively $-5.24 \times 10^{-7} \pm 1.39 \times 10^{-6} \mathrm{~s}^{-1},-7.45 \times 10^{-7} \pm 1.64 \times 10^{-6} \mathrm{~s}^{-1}$, and $-1.74 \times 10^{-6} \pm 1.58 \times$ $10^{-6} \mathrm{~s}^{-1}$. 


\section{Discussion and Conclusion}

\subsection{Peak characterization}

Let us first look at the vacuum data and try to identify the peaks there. We can see from Figure $3 \mathrm{~b}$ that the peak at $1.345 \mathrm{~V}$ is well isolated and has the highest energy; this appears to correspond to the ${ }^{212} \mathrm{Po} \alpha$ decay energy, which at $8.78 \mathrm{MeV}$ is the highest energy in the decay scheme and is also well separated from the other energies (see Figure 1). On the other hand, assuming that the number of ${ }^{228} \mathrm{Th}$ decays detected is negligible due to the relative positioning of the source and the detector (see Section 2), the lowest $\alpha$ decay energy in the scheme is that of ${ }^{224} \mathrm{Ra}$, at $5.67 \mathrm{MeV}$. We therefore hypothesize that the peak with the lowest energy, at $0.859 \mathrm{~V}$, represents ${ }^{224} \mathrm{Ra} \alpha$ decays. Assuming that our highest and lowest energies are represented by our highest and lowest pulse heights, we linearly calibrate the other four alpha decay energies in the scheme to obtain four predicted pulse voltages, displayed in Table 1. Comparing the pulse voltage predicted by calibration for ${ }^{220} \mathrm{Rn}$ decays $(0.956$ $\mathrm{V})$, and the voltage at which we observed the second peak $(0.957 \mathrm{~V})$, we find that their difference is small, much smaller than the $\sigma$ value of $0.022 \mathrm{~V}$ obtained from the Gaussian fit for the second peak. Therefore it appears that the second peak represents the energies from

${ }^{220} \mathrm{Rn}$ decays. Performing the same analysis, we find a similar correspondence between ${ }^{216} \mathrm{Po}$ decays and the third peak also. Similarly, the two possible $\alpha$ decays of ${ }^{212} \mathrm{Bi}$ both seem to correspond to the apparent shoulder between the first and second peaks (see Figure 3c), the differences between the calibrated pulse voltages and the observed mean again well within the $\sigma$ value. We also observe that the two predicted pulse voltages differ by only $0.006 \mathrm{~V}$, about half of $\sigma$. With such a small difference, it seems that we cannot distinguish between the two energies with our data. The shoulder we see between the second and third peaks appears to represent a combination of the two possible $\alpha$ decays from ${ }^{212} \mathrm{Bi}$.

Thus we are able to account for all the peaks and the shoulder observed in the vacuum 
data and determine their corresponding $\alpha$ decays (see Figure 3d). Since our assumption that the first peak represents a peak of ${ }^{224}$ Ra decays has resulted in a good calibration, we conclude that in a vacuum, the average pulse signal given by ${ }^{224}$ Ra decays is approximately $0.86 \mathrm{~V}$, with the subsequent decays of ${ }^{212} \mathrm{Bi},{ }^{220} \mathrm{Rn},{ }^{216} \mathrm{Po}$, and ${ }^{212} \mathrm{Po}$ giving pulse signals of $0.92 \mathrm{~V}, 0.96 \mathrm{~V}, 1.04 \mathrm{~V}$, and $1.34 \mathrm{~V}$, respectively.

Unfortunately, the xenon gas data are much more complicated and elude a similarly straightforward analysis. First, as mentioned in Section 2, we used a different pre-amplifier for the background counts in xenon gas, which might have had an effect on the size of the pulse signals. This seems to be have been the case, as Figure $5 \mathrm{c}$, with the vacuum background histogram drawn in black and the xenon gas histogram (at source position) drawn in red, shows that the peaks for the background in xenon gas have shifted higher. We attribute this increase to the pre-amplifier switch, because normally we would expect the peaks to shift down, as alpha particles should lose rather than gain energy in gas. With the shifted peaks, we cannot do a comparison of the absolute values as we had hoped to do. We attempted to resort to comparing the differences between peaks - for instance, the difference between the two rightmost peaks in vacuum is $0.306 \mathrm{~V}$, and in xenon gas, $0.334 \mathrm{~V}$ - and see how they compare with the values predicted from the stopping power of alpha particles in xenon (according to [3]) and the density of xenon at 0.5 atm (according to [4]), but this approach did not work very well. The reason for this may be that the spectrum we are looking at is really much more complicated, with many peaks hidden inside. We can observe from Figure $5 \mathrm{c}$ that except for the rightmost peak, the peaks in the xenon gas histogram are not as well-defined as they are in the vacuum histogram, particularly in the lower range, which seem to have a combination of several peaks that are very difficult to differentiate. These peaks may be due to that in xenon gas, the particles accepted by the detector came from different parts of the chamber - some have traveled longer distances, and others shorter, so the energies they have lost to the gas might have been significantly different as well. This 
would break up the well-defined peaks observed in vacuum and result in smaller peaks at points we would not expect. In the end we conclude that we cannot characterize the $\alpha$ spectrum with so many ambiguities and complications. Perhaps in the future, by making a vacuum background count with the new amplifier, we can make a more accurate analysis.

\subsection{Time decay}

We are also interested in the decay rates that were observed. From Figure 4, we see that all four slopes from the exponential fits for our vacuum data are quite flat, with two of them positive and the other two negative. Of course, it is nonphysical to have a positive decay rate for a decaying element. However, we also see that all four slopes have relatively large errors, with considerable scattering in the data, and in each case, 0 lies within $2 \sigma$ s from the mean, so even in the cases of positive slopes, the actual value may well be a very small negative value. Since the slopes are relatively flat and the data seem to scatter around a constant level, we can reasonably say that the actual slope for the data is very close to zero, and that the decay rates observed in vacuum were very low, in accordance with ${ }^{228}$ Th's long half-life of 1.9 years.

We observe similar results with the xenon gas background counts: the slopes are negative and very small, with errors that are larger or almost as large as the slopes. This is consistent with the decay rates being very nearly zero, due to ${ }^{228}$ Th's half-life. This is actually not the result we expected - we anticipated the decay rate in xenon gas to follow that of ${ }^{224} \mathrm{Ra}$, because the reversed-biased source should have attracted back any Ra ions that decayed from the Th, so that the decays seen by the detector are only those from Ra atoms (and their daughter nuclei) already deposited on different parts of the chamber before the count was initiated. This then would result in the decay rate being determined by Ra, rather than Th. One possible explanation for the Th decay rate that we are observing is that a significant number of Ra ions recoiled from the source might have recombined quickly with electrons to become neutral again. 


\section{Acknowledgements}

This research was conducted at the Stanford Linear Accelerator Center. I would like to take this opportunity to thank my mentor Peter Rowson for his guidance and support throughout the past weeks. I am also grateful for the help and enthusiasm from everyone else in EXO at SLAC. In particular, I would like to thank Kolo Wamba for his day-to-day guidance and patience. Finally, I thank the U. S. Department of Energy, Office of Science, and SLAC for organizing and funding the SULI program and making this tremendous learning experience possible. 


\section{Tables and Figures}

\begin{tabular}{llllll}
\hline \hline Element & $E(\mathrm{MeV})$ & Predicted $V(\mathrm{~V})$ & Observed $V(\mathrm{~V})$ & $\Delta V(\mathrm{~V})$ & $\sigma_{V}(\mathrm{~V})$ \\
\hline${ }^{224} \mathrm{Ra}$ & 5.67 & $(0.859)$ & 0.859 & $(0)$ & 0.023 \\
${ }^{212} \mathrm{Bi}(25 \%)$ & 6.05 & 0.919 & 0.922 & 0.003 & 0.012 \\
${ }^{212} \mathrm{Bi}(10 \%)$ & 6.09 & 0.925 & 0.922 & -0.003 & 0.012 \\
${ }^{220} \mathrm{Rn}$ & 6.29 & 0.956 & 0.957 & 0.001 & 0.022 \\
${ }^{216} \mathrm{Po}$ & 6.78 & 1.032 & 1.039 & 0.007 & 0.018 \\
${ }^{212} \mathrm{Po}$ & 8.78 & $(1.345)$ & 1.345 & $(0)$ & 0.022 \\
\hline \hline
\end{tabular}

Table 1: Decay Energy $(E)$-Pulse Voltage $(V)$ Calibration

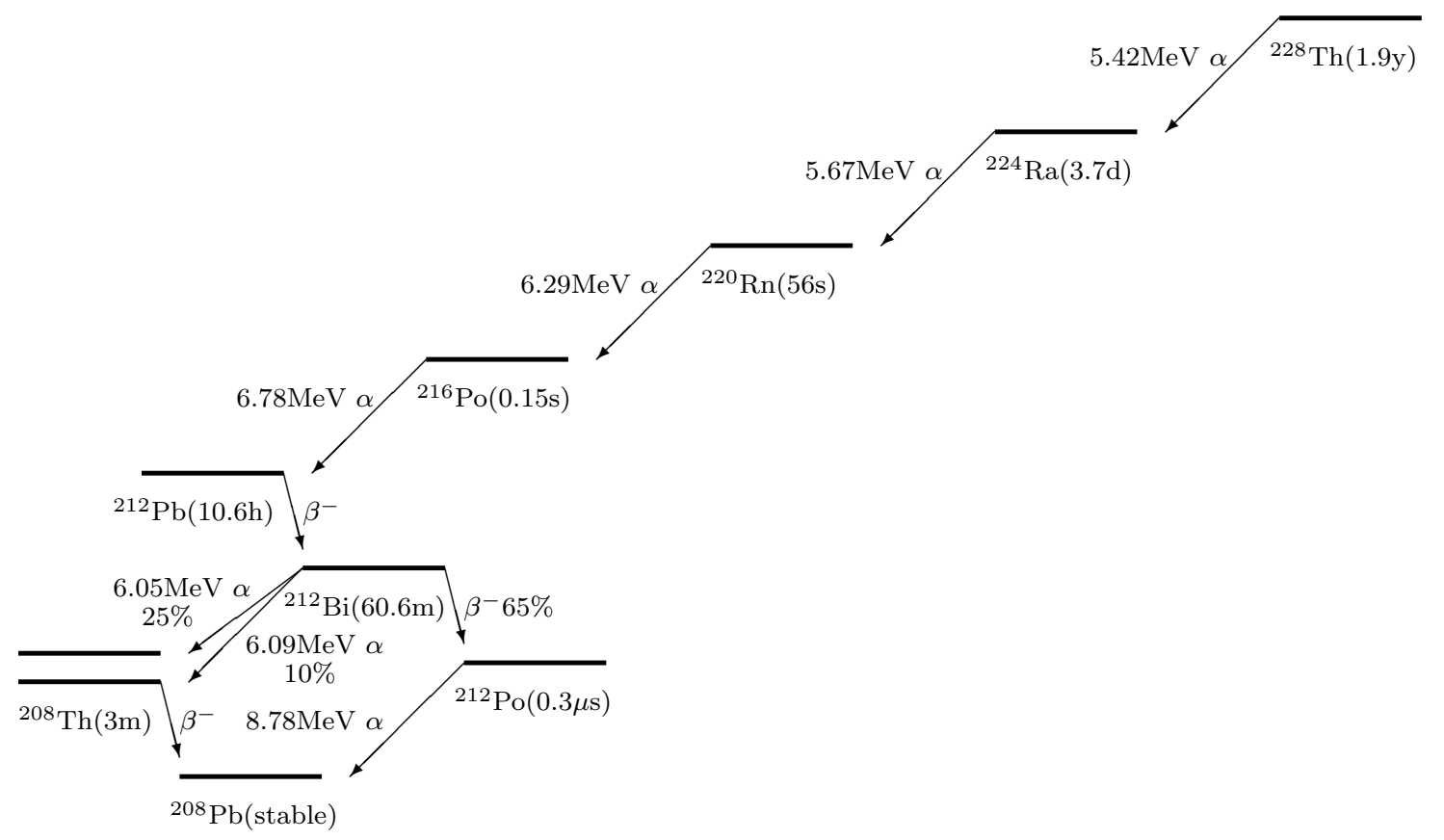

Figure 1: Level scheme for ${ }^{228} \mathrm{Th}$. 


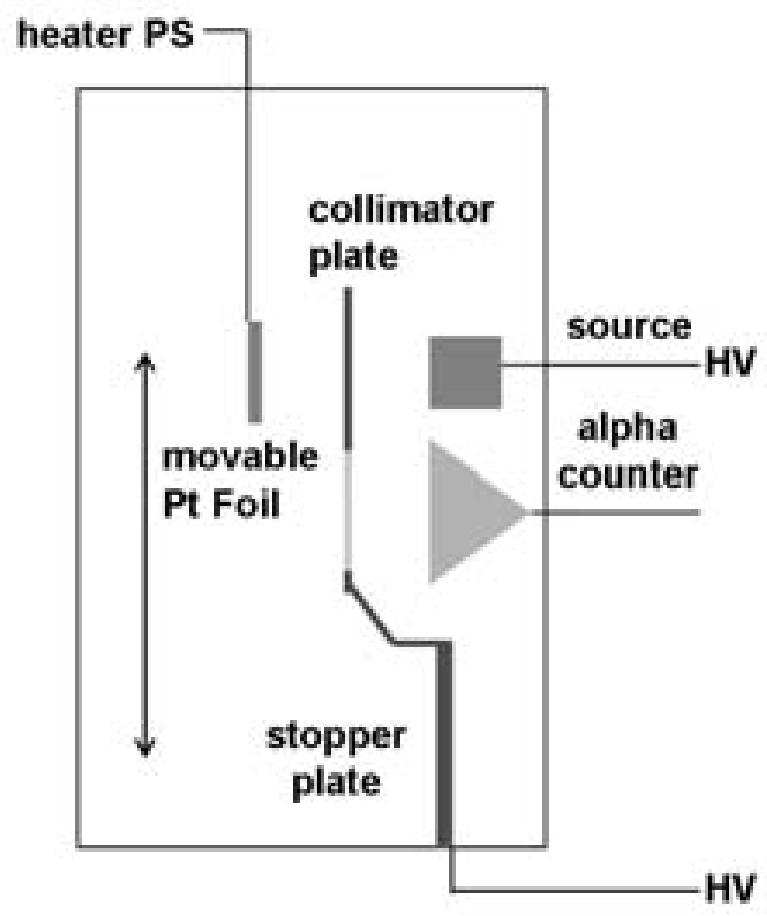

Figure 2: Diagram of the apparatus for the experiment. 


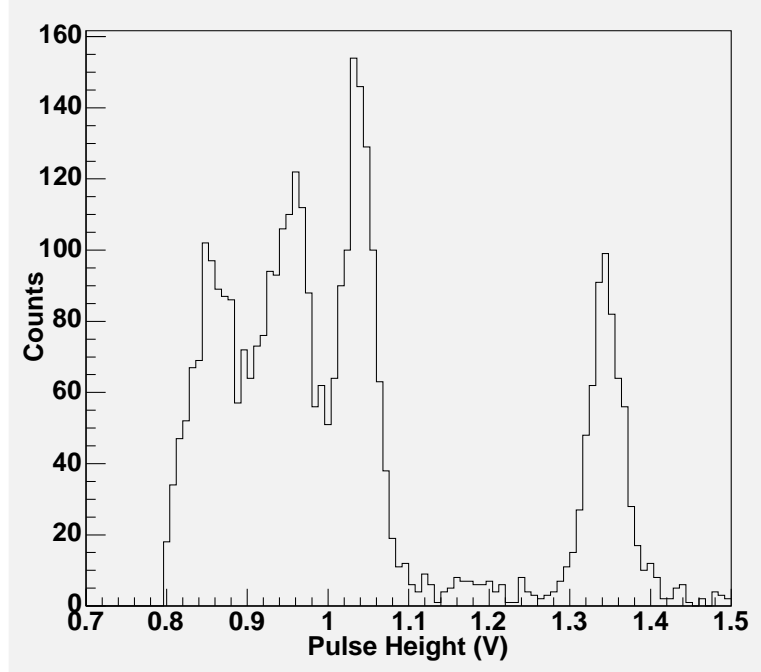

(a)

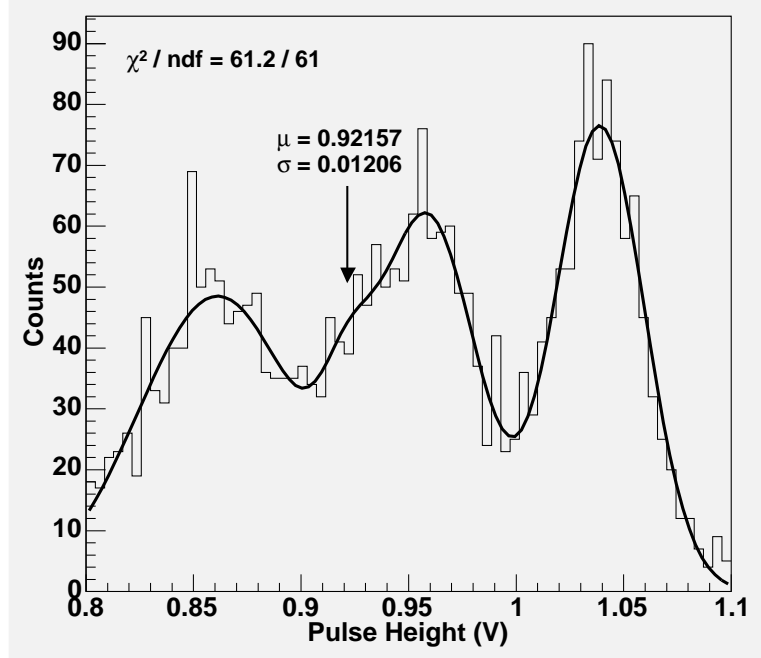

(c)

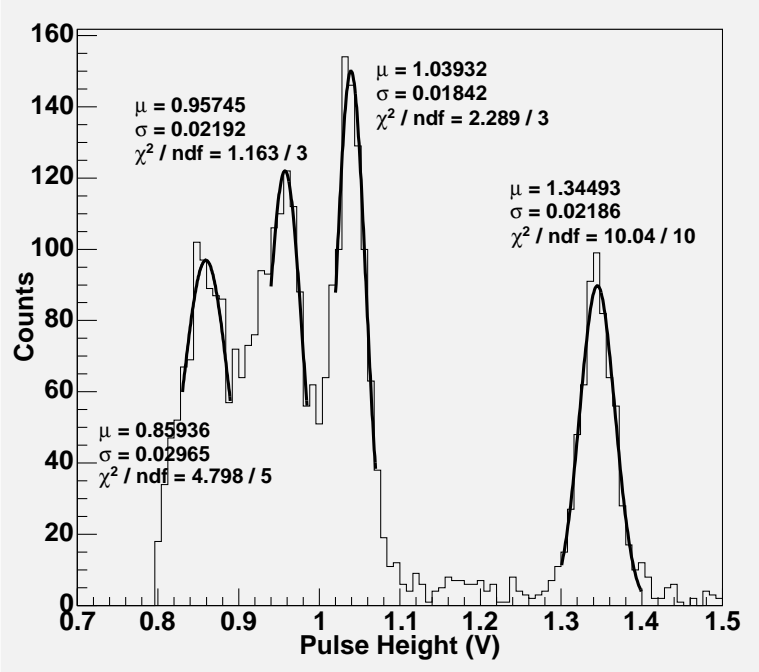

(b)

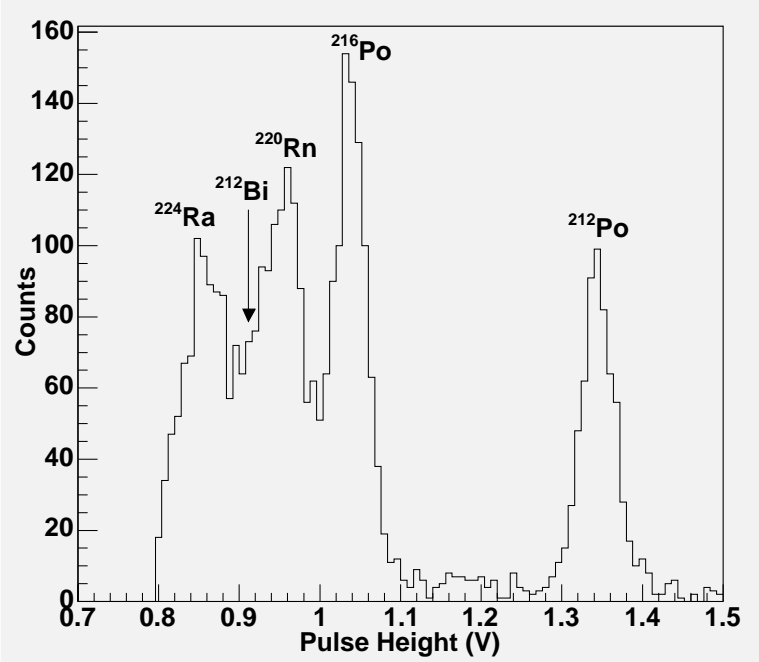

(d)

Figure 3: (a): Histogram of pulses observed in vacuum. (b): A Gaussian curve is fitted for each peak. (c): A sum of four Gaussian curves is fitted over a smaller range, for the first three peaks and the apparent shoulder between the two leftmost peaks. (d): The spectrum is labeled with corresponding decaying elements. 

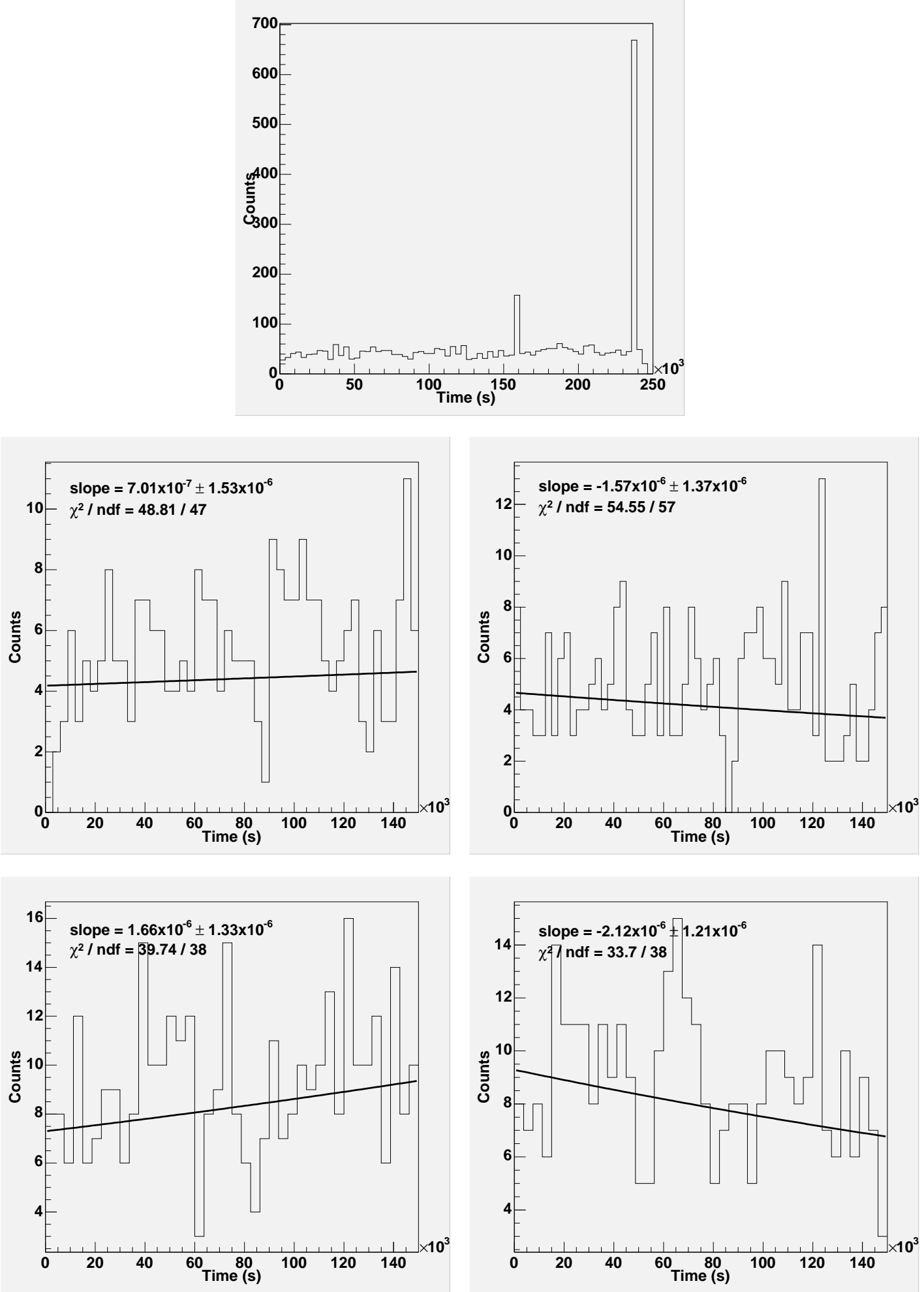

Figure 4: Histogram of times at which particular ranges of pulses were observed, in vacuum. Top: The entire range, $0.8-1.5 \mathrm{~V}$. Middle left: ${ }^{224} \mathrm{Ra}$ range, $0.83-0.89 \mathrm{~V}$. Middle right: ${ }^{220} \mathrm{Rn}$ range, $0.94-0.985 \mathrm{~V}$. Bottom left: ${ }^{216} \mathrm{Po}$ range, $1.02-1.07 \mathrm{~V}$. Bottom right: ${ }^{212} \mathrm{Po}$ range, $1.3-1.4 \mathrm{~V}$. 


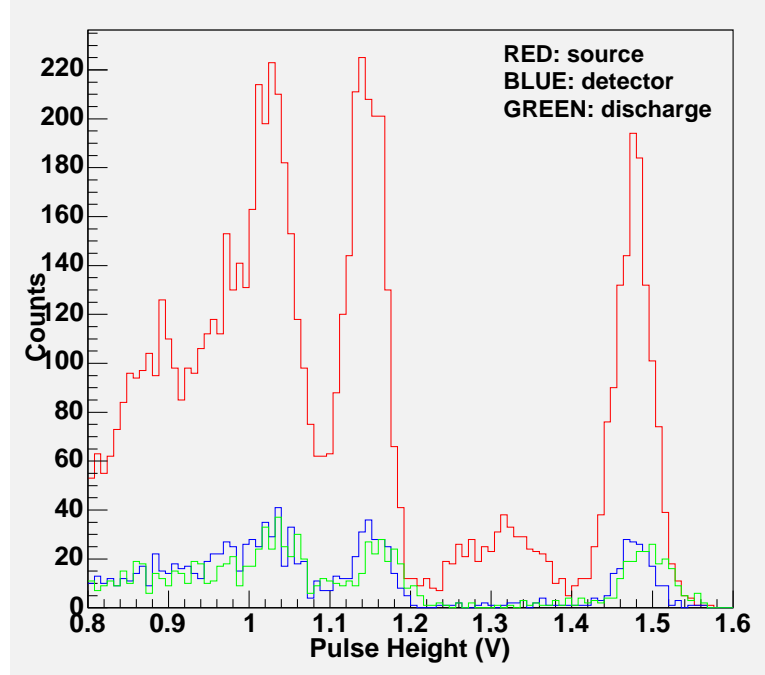

(a)

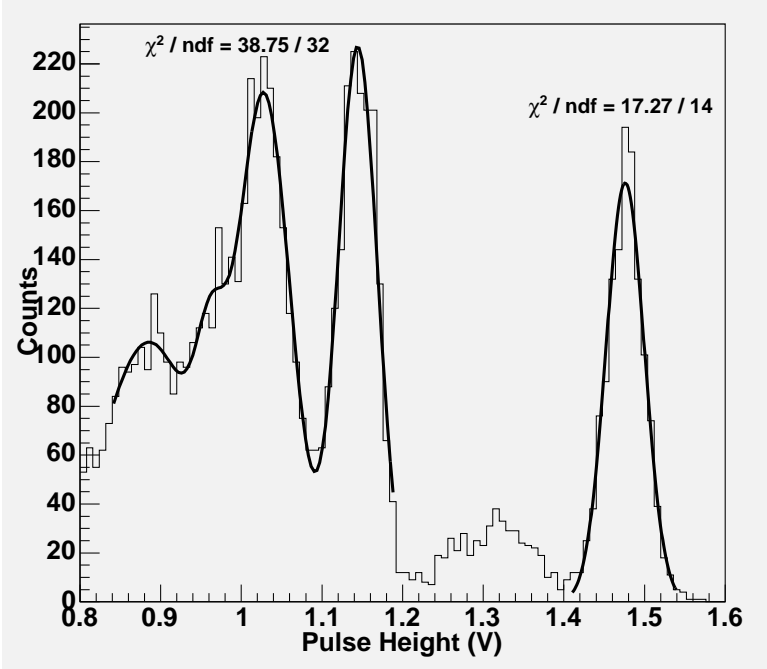

(b)

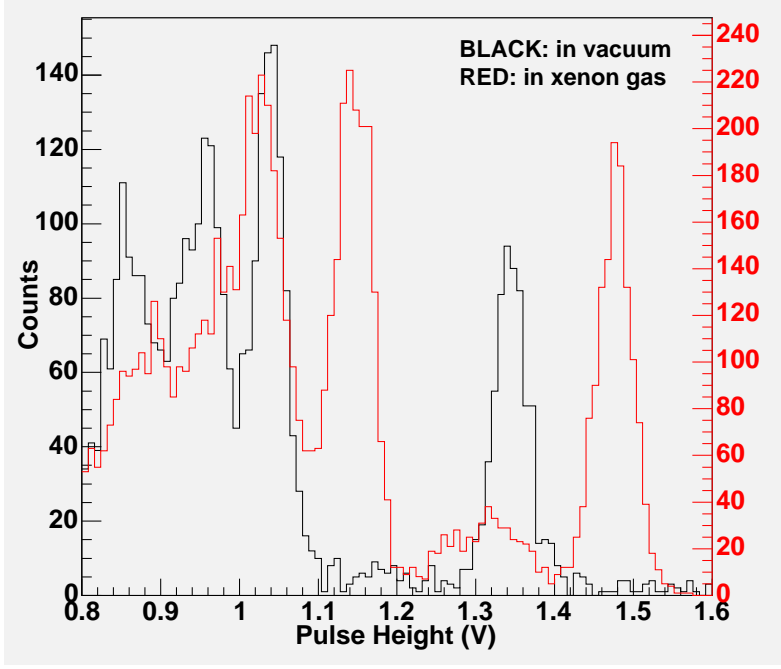

(c)

Figure 5: (a): Superimposition of the histograms for the three background counts done with the chamber filled with xenon gas; the foil is at a different position for each count, as indicated by the colors. (b): Histogram for the 72-hour count at source position, with Gaussian curve fits. (c): Scaled superimposition of the vacuum background histogram and the "source" xenon gas histogram. 

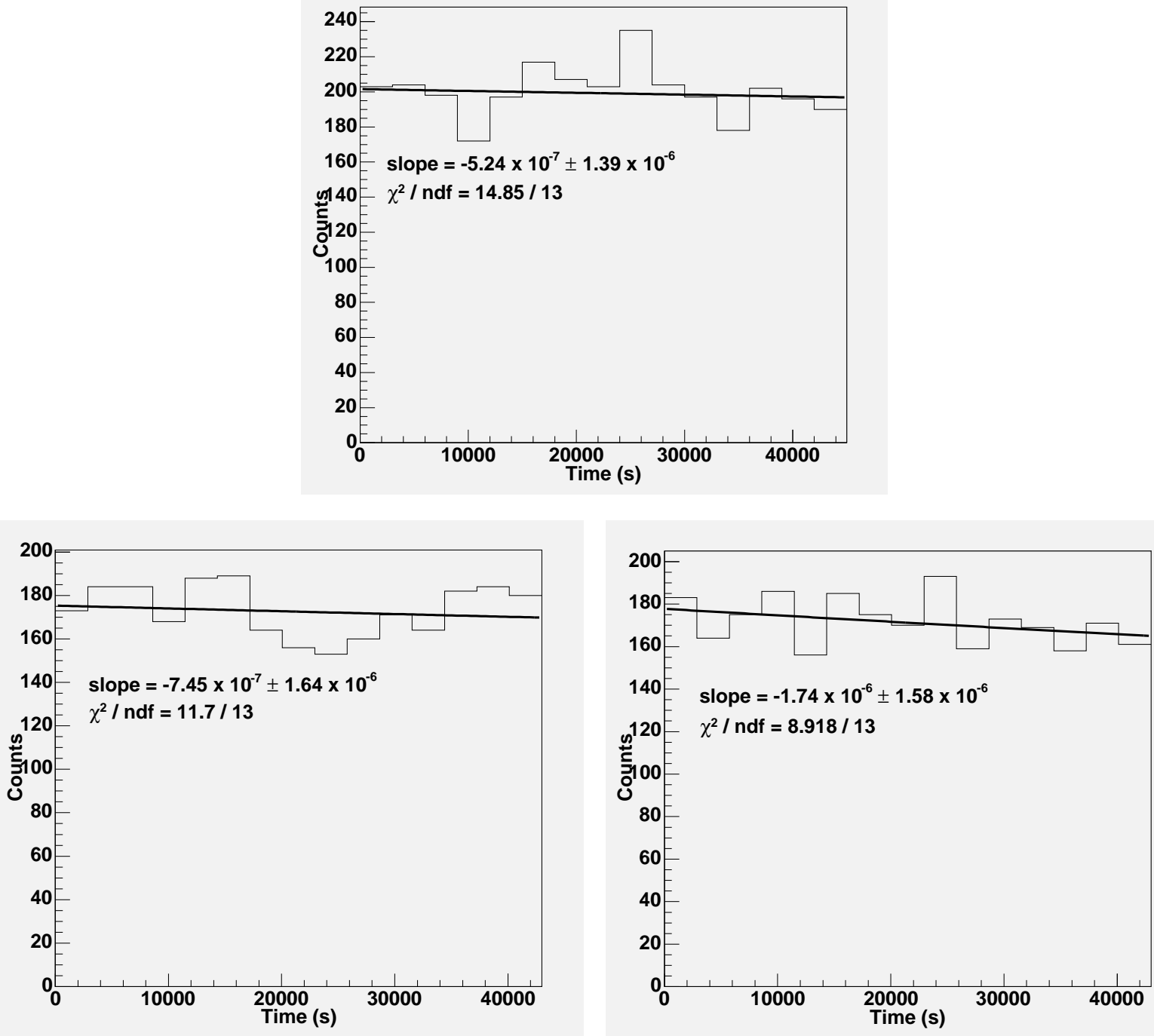

Figure 6: Time histograms for xenon gas background. Top: Source position. Bottom left: Detector position. Bottom right: Discharge position. 


\section{References}

[1] P.C. Rowson, "Barium Ion Tagging: Ion Acquisition in LXe \& Laser Fluorescence Identification," presented at EXO Week, Stanford, CA, 2005.

[2] K. Wamba, "Proposal for Ion Grabbing Experiments with a ${ }^{228}$ Th Source," EXO Collaboration ELOG, no. 36 .

[3] M.J. Berger, J.S. Coursey, M.A. Zucker, and J. Chang, ESTAR, PSTAR, and ASTAR: Computer Programs for Calculating Stopping-Power and Range Tables for Electrons, Protons, and Helium Ions, [Online database] Gaithersburg, MD: National Institute of Standards and Technology, [2005 Aug 17], Available at HTTP: http://physics.nist.gov/Star

[4] E.W. Lemmon, M.O. McLinden and D.G. Friend, "Thermophysical Properties of Fluid Systems" in NIST Chemistry WebBook, NIST Standard Reference Database Number 69, [Online database] Eds. P.J. Linstrom and W.G. Mallard, Gaithersburg, MD: National Institute of Standards and Technology, June 2005, [2005 Aug 17], Available at HTTP: http://webbook.nist.gov 